\title{
Journal of

\section{Template free preparation of nanoporous organically modified silica thin films on flexible substrates $\dagger$}

\author{
Adem Yildirim, ${ }^{a b}$ Hulya Budunoglu, ${ }^{a b}$ Mecit Yaman, ${ }^{a b}$ Mustafa O. Guler ${ }^{a b}$ and Mehmet Bayindir ${ }^{* a b c}$ \\ Received 23rd May 2011, Accepted 21st July 2011 \\ DOI: 10.1039/c1jm12188j
}

\begin{abstract}
We report the preparation and characterization of nanoporous organically modified silica (ormosil) thin films at room temperature and neutral $\mathrm{pH}$ conditions from homogeneous methyl silsesquioxane (MSQ) gels. Universally applicable and stable colloidal ormosil suspensions are prepared from the gels by sonication and coated to the substrates including glass, paper and plastics. The nanoporosity and thickness of the films can be tuned, which makes them suitable for certain applications including sensing, functional coatings, and low-dielectric materials. We demonstrate the antireflection property of the films on glass, cellulose acetate (CA) and polyetherimide (PEI) substrates. The films on CA and PEI retain their antireflection property after multiple bending cycles. Furthermore, films are intrinsically hydrophobic, over a wide $\mathrm{pH}$ range, with static contact angles up to $143^{\circ}$ on paper and $123^{\circ}$ on glass and CA. Producing nanoporous ormosil thin films on flexible substrates may expand their use in low cost electronic, optical devices and sensors, and lab-on-paper applications.
\end{abstract}

\section{Introduction}

Organically modified silica (ormosil) hybrids offer both organic polymers' mechanical flexibility and inorganic polymers' durability. ${ }^{1,2}$ In ormosils, organic moieties covalently bonded to a durable silica network provide the flexibility by decreasing cross-linking of the network. As a result of this unique property, they are ideal materials for anticorrosion, antifouling and fire retardation applications. ${ }^{3-8}$ Furthermore, nanoporosity is added to ormosils in order to provide extra-functionalities such as high surface area ${ }^{9-11}$ and therefore adsorption capacity, ${ }^{12,13}$ and very low refractive index ${ }^{14,15}$ and dielectric constant. ${ }^{16}$ Nanoporous ormosil bulk materials and thin films can be prepared using selfassembling template molecules to form periodic nanopores ${ }^{17}$ or by sacrificial polymers to induce nano-phase separation. ${ }^{18}$ However, the additional removal step of sacrificial substances requires harsh conditions, i.e. high temperature $\left(400-450{ }^{\circ} \mathrm{C}\right)$ and/or low $\mathrm{pH}(<1)$, further complicating the process, and limiting the choice of substrate in the case of thin films. High temperature and acid treatments limit the choice of substrates such as glass or silicon, which can resist these conditions. Substrates such as paper and plastics, which are extensively used in fabrication of flexible low-cost electronic and photovoltaic devices, cannot survive under such extreme conditions. ${ }^{19-21}$ Thus,

${ }^{a}$ UNAM-National Nanotechnology Research Center, Bilkent University, 06800 Ankara, Turkey. E-mail: bayindir@nano.org.tr

${ }^{b}$ Institute of Materials Science and Nanotechnology, Bilkent University, 06800 Ankara, Turkey

${ }^{c}$ Department of Physics, Bilkent University, 06800 Ankara, Turkey

$\dagger$ Electronic supplementary information (ESI) available. See DOI: $10.1039 / \mathrm{c} 1 \mathrm{jm} 12188 \mathrm{j}$ new fabrication methods under mild conditions for nanoporous ormosils are strongly desired.

In this context, the promise of template free methods is the production, by eliminating the harsh template removal steps, of nanoporous ormosil thin films at room temperature and neutral pH conditions. Although, Prakash et al. ${ }^{22}$ were able to prepare porous silica thin films under mild conditions from colloidal suspensions of surface modified silica gels, these films prove to be inflexible because of the rigid silica network. Also, our previous work $^{23}$ reports porous thin films from phase separated methyl silsesquioxane (MSQ) gels. In MSQ gels, phase separation occurs due to the low solubility of cyclic and cage like structures formed during polymerization. ${ }^{24}$ Although, these films are flexible because of the organically modified silica network, non-uniform film formation and macroporous structure are observed because of phase separation. So, to prepare uniform, flexible, and nanoporous thin films from silica based gels, homogeneous gels including organic moieties directly bound to the silica network must be prepared. For this purpose, we synthesized new methyl silsesquioxane (MSQ) gels by using methyltrimethoxysilane (MTMS) as precursor and dimethyl sulfoxide (DMSO) as solvent. The gels are surprisingly homogeneous instead of the commonly obtained macroscopic phase separated MSQ gels when prepared in alcohols by using the same precursor. ${ }^{25-27}$ This is because all structures formed during polymerization are dissolved in DMSO. Here, for the first time, we prepared uniform and nanoporous thin films by using homogeneous MSQ gels.

The homogeneous MSQ gels are broken down by sonication to obtain colloidal suspensions which are suitable for thin film deposition. The prepared ormosil suspensions are found to be stable in solution without sedimentation for several months. 
These colloidally stable ormosil suspensions offer easy handling and good reproducibility for large-area fabrication of uniform nanoporous ormosil thin films on almost any substrate including plastics, paper, glass and silicon by common thin film deposition methods such as spin, dip and spray coating. The method enables preparation of homogeneous films as thin as $90 \mathrm{~nm}$. In addition, porosity (therefore the refractive index) of the films can be controlled by simply adjusting the monomer concentration in the starting solution. Controllable nanoporosity and thickness pave the way for certain applications including support materials for sensors and catalysts, antireflective coatings for photovoltaics, and low-dielectric constant films for microelectronics. In this work we present a detailed investigation of nanoporous ormosil thin films as antireflective coatings. We characterized the antireflective properties of the films on glass, cellulose acetate (CA) and polyetherimide (PEI) substrates. The films exhibit mechanically durable antireflective performance after several cycles of bending on flexible substrates. Furthermore, films are intrinsically hydrophobic increasing the durability of the pore structure in atmospheric conditions, and the hydrophobicity of the films is not affected by the environmental $\mathrm{pH}$.

\section{Experimental}

\section{Materials}

Methanol, DMSO, ammonium hydroxide, oxalic acid and methyltrimethoxysilane (MTMS) were purchased from SigmaAldrich (USA). All of the chemicals were used as received.

\section{Gel preparation}

The gels were named, with the first letter in the abbreviation (D7) indicating the solvent type, 'D' for DMSO and ' $\mathrm{M}$ ' for methanol, and the numerical indicating the solvent/MTMS ratio (v/v).

Gels were prepared by a two step acid-base catalysis mechanism by modifying the previous methods. ${ }^{23,27,29}$ In the first step, $1 \mathrm{ml}$ of MTMS was dissolved in appropriate amounts of DMSO or methanol. For example, for the D7 gel a total of $7 \mathrm{ml} \mathrm{DMSO}$ was used. First $1 \mathrm{ml}$ MTMS was dissolved in $2 \mathrm{ml}$ DMSO. Then $0.5 \mathrm{ml}$ of $10 \mathrm{mM}$ oxalic acid solution in water was added slowly and the solution was stirred for 30 minutes. After that, $0.45 \mathrm{ml}$ ammonia solution $(26 \%)$ and $0.2 \mathrm{ml}$ water in $5 \mathrm{ml}$ DMSO were added and the solution was stirred for $15 \mathrm{~min}$ at room temperature. For other gels only the amount of solvent in the second solution was changed to adjust the total solvent amount. The solution was then allowed for gelation. Gels were formed typically in one hour. Prepared gels were washed 4 times with methanol. Each washing step lasted at least 6 hours. An appropriate aging time is crucial for strengthening the gel network and preventing pore collapse during sonication and solvent evaporation. ${ }^{28,29}$ Here, gels were aged during the washing steps and this was found to be sufficient to obtain highly porous colloids and thin films.

\section{Colloidal suspension preparation}

Washed gels were broken into small pieces and the gel volume was doubled with methanol. After that, it was sonicated with an ultrasonic homogenizer to obtain a suitable sol for film preparation. The optimum time and power for homogenization were found to be $45 \mathrm{~s}$ and $20 \mathrm{~W}$.

\section{Film preparation}

A spin-coating method was used for thin film production. The suspension was spin-coated on substrates at $2000 \mathrm{rpm}$. The substrates were sonicated in ethanol or rinsed with isopropanol before coating. Most of the solvent evaporates during the spincoating process. The films were dried at room temperature overnight for further solvent evaporation.

\section{Characterization}

ESEM and SEM images were taken with a FEI Quanta 200F ESEM microscope at low vacuum conditions. TEM bright-field images were taken using a FEI Tecnai G2 F30 microscope operated at $200 \mathrm{kV}$. TEM samples were prepared by diluting the suspensions in methanol. A drop of the solution was placed on a holey carbon-coated copper grid. The surface roughness was analyzed using an atomic force microscope (AFM, XE-100E, PSIA) in non-contact mode. UV-vis transmission spectra were recorded by a Varian Carry100 spectrophotometer. Thickness and refractive index measurements were performed by using a $\mathbf{J}$. A. Woollam V-Vase Ellipsometer. A contact angle meter (OCA 30, Dataphysics) was used to measure the static water contact angles on the aerogel thin films. Water droplets of $0.4 \mu \mathrm{L}$ volume were used and Laplace-Young fitting was applied on contact angle measurements. Functional groups of surfaces were characterized by using a Fourier transform infrared spectrometer (FTIR, Vertex 70, Bruker) and an X-ray photoelectron spectrometer (XPS, K-Alpha, Thermo Scientific).

\section{Results and discussion}

\section{Preparation of transparent MSQ gels}

In this work, we first prepared transparent MSQ gels by using only methyltrimethoxysilane (MTMS) as monomer. Gelation of MTMS in alcohols results in macroscopic phase separation, due to low solubility of cyclic and cage like structures formed during polymerization of this trifunctional monomer. In our previous works we reported that thin films produced from such macroscopic phase separated gels have translucent appearance and for the films with thicknesses lower than $500 \mathrm{~nm}$ non-uniform film formation is observed due to macroporosity. ${ }^{23,29}$ Thus, to produce uniform nanoporous thin films, the macroscopic phase separation in the MSQ gels should be prevented. Kanamori et al. ${ }^{24}$ prepared highly transparent MSQ gels (indicating no or little phase separation occurred) by using different surfactant solutions in water. Here, we utilize a novel strategy to prevent phase separation in MSQ by using DMSO as solvent (Scheme 1). Briefly, MTMS is dissolved in an appropriate amount of DMSO and oxalic acid is added for hydrolysis. Then for condensation reaction ammonia is added with water. Gels are formed within one hour depending on the MTMS concentration, and longer gelation times were observed for lower MTMS concentrations. We observed that using DMSO as solvent instead of alcohols resulted in homogeneous and transparent MSQ gels most probably by dissolving the cyclic and cage like structures forming 


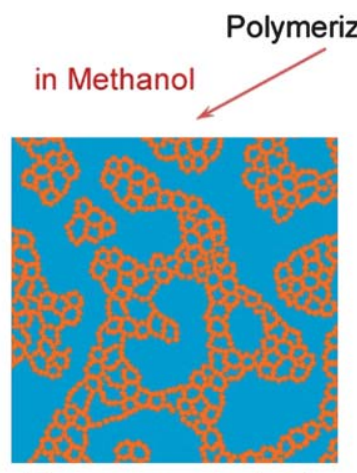

Phase-separated gel in DMSO

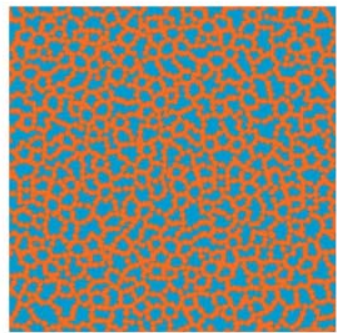

Homogenous gel

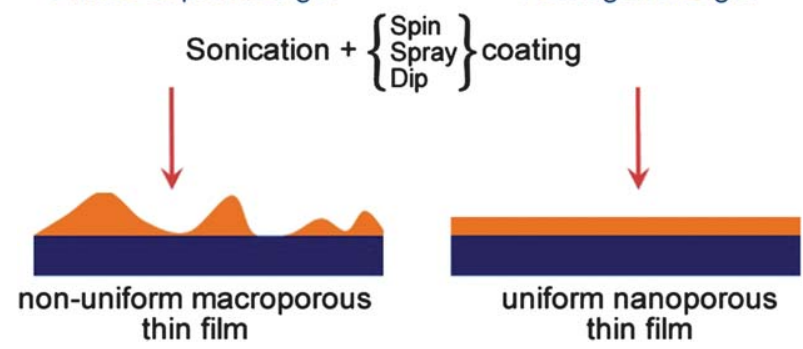

Scheme 1 Schematic representation of gels and their thin films. When MTMS polymerized in methanol phase separation occurs, on the other hand, when polymerized in DMSO homogeneous gels are obtained. Uniform and nanoporous thin films can be prepared by using these homogeneous gels.

during polymerization (Fig. 1a). On the other hand, the gels prepared in methanol, at same conditions, are opaque because of phase separation (Fig. 1b).

Structures of the gels are investigated with environmental scanning electron microscopy (ESEM) (Fig. 1c and d). The ESEM image of the gel prepared in methanol demonstrates rough structure consisting of micrometre sized particles indicating phase separation, which is in accordance with a previous report. ${ }^{26}$ On the other hand, the ESEM image of the gel prepared by using DMSO as solvent shows smooth surface (gel network was further characterized with SEM and TEM after drying and given below) indicating homogeneous gel formation without phase separation. Homogeneous MSQ gel formation in DMSO demonstrates that all species formed during polymerization can effectively dissolve in the system. Also the gelation time of MTMS in DMSO is shorter than that of methanol at the same conditions. DMSO is more polar than methanol which may accelerate the hydrolysis and condensation reactions yielding faster polymerization of MTMS.

\section{Preparation of stable colloidal ormosil suspensions}

Prepared MSQ gels were first washed with methanol to exchange the gel solvent. After washing, gels were sonicated to form colloidal ormosil suspensions by breaking down the gel network. It was observed that solvent exchange with methanol is crucial for preparation of stable suspensions. The colloidal suspensions prepared without the solvent exchange process polymerize and form gels within a day because the base in the suspension facilitates co-condensation reactions. By methanol washing, a stable suspension can be achieved since the base is removed. It is also
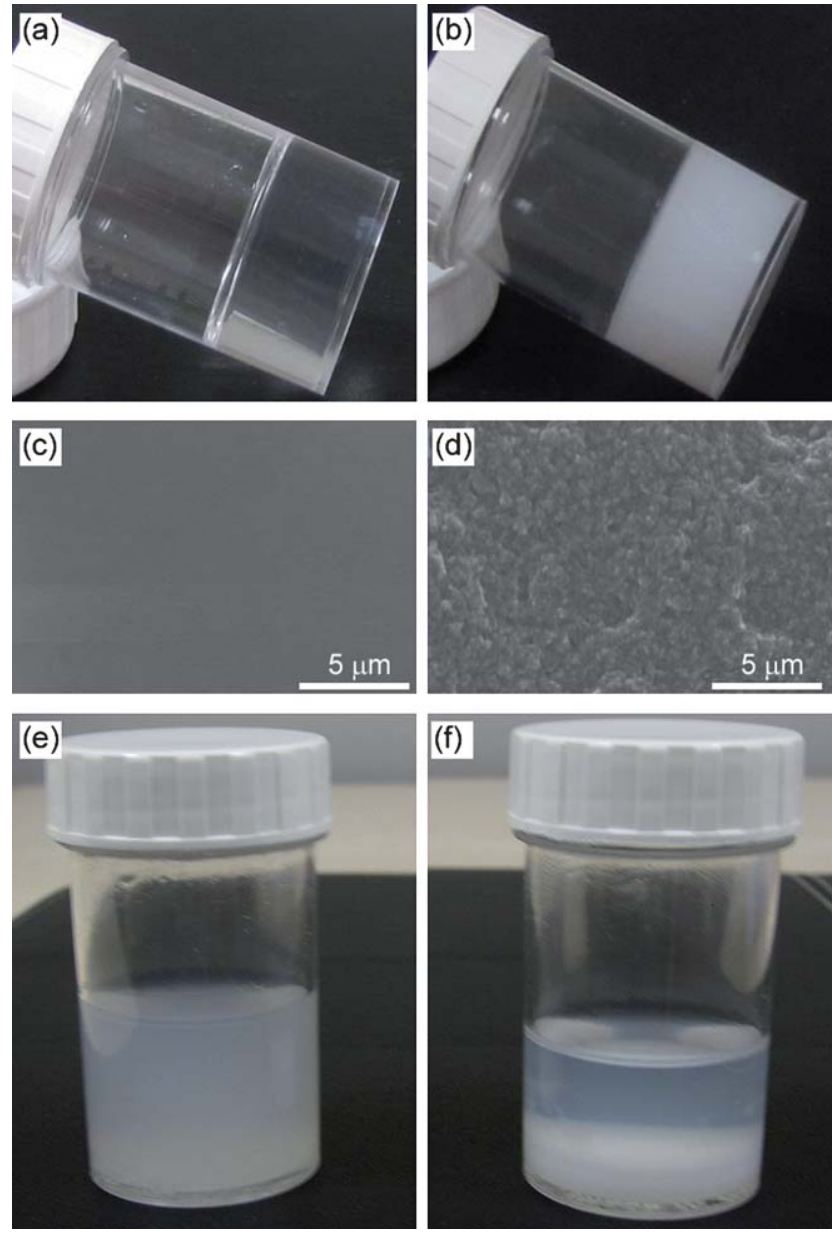

Fig. 1 Photographs of gels and colloidal suspensions. Gel prepared (a) in DMSO and (b) in methanol. The gel prepared in DMSO is transparent indicating homogeneous gel formation. On the other hand, the gel prepared in methanol is opaque because of phase separation. ESEM micrographs of (c) homogeneous and (d) phase-separated gels. (e) Colloidal suspension of the gel in DMSO, and (f) the gel in methanol. Colloidal suspension of the gel prepared in DMSO is stable even after 3 months whereas for methanol it precipitates in a day.

noted that other low molecular weight alcohols such as ethanol and isopropanol can be used for the solvent exchange process.

Suspensions prepared from MSQ gels prepared in DMSO are colloidally stable (up to 3 months at $4{ }^{\circ} \mathrm{C}$ without significant agglomeration shown in Fig. 1e), universally applicable and nanoporous. Stability of the colloidal ormosil suspensions stems from inert methyl groups of the MTMS monomer, which covers the particle surface and prevents particle aggregation. ${ }^{30}$ However, suspensions of MSQ gels prepared in methanol are precipitated in a day (Fig. 1f). This may be because ormosil particles in these suspensions are bigger than those in the suspensions prepared from gels synthesized by using DMSO as solvent (Fig. $2 \mathrm{a}$ and b), so that they precipitate easily.

In this work, several ormosil suspensions were prepared from MSQ gels with different solvent/MTMS (v/v) ratios ranging from 4 to 15 . The structure of ormosil suspensions was investigated by using a Transmission Electron Microscope (TEM) (Fig. 2). The D7 suspension reveals a nanoporous structure of interconnected 

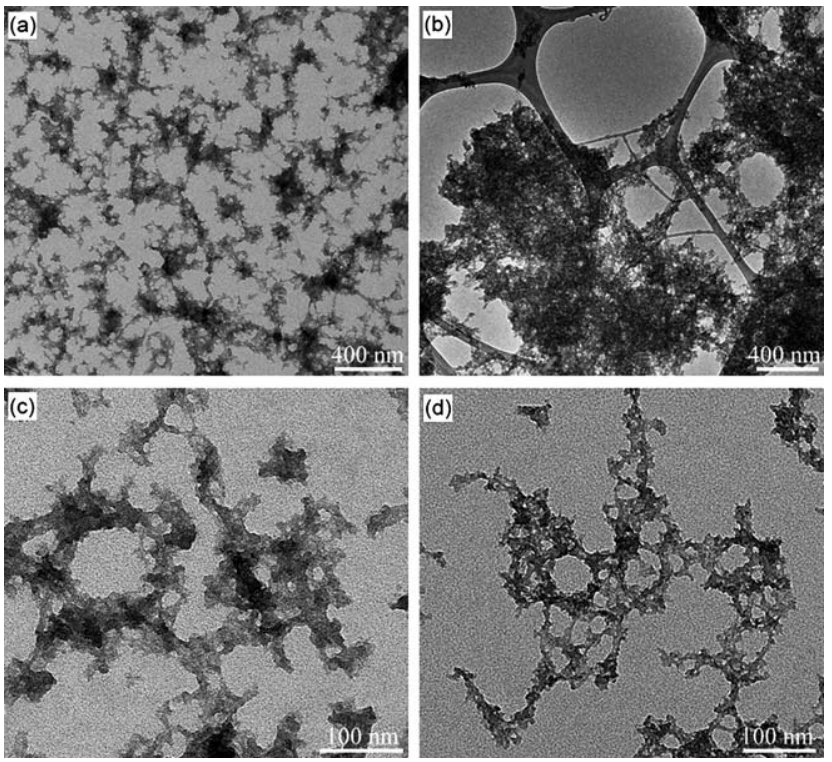

Fig. 2 TEM images of suspensions. (a, c) D7 suspension, (b) M7 suspension and (d) D15 suspension. Approximately $20 \mathrm{~nm}$ sized particles interconnect and form mesoporous structure. The particles in the M7 suspension are larger than both in D7 and D15 suspensions. The D15 suspension exhibits more porous structure with thinner walls compared to the D7.

ormosil particles (Fig. 2a-c). According to these images, nanoparticles smaller than $50 \mathrm{~nm}$ interconnect and form a porous structure. The D15 reveals a more porous structure with thinner walls compared to the D7 (Fig. 2d). The more porous structure of D15 is due to the lower MTMS concentration of the gel used to prepare this suspension.

\section{Preparation of nanoporous ormosil films}

Ormosil colloidal suspensions were coated on various surfaces by the spin coating method. Fig. 3 shows the morphologies of spin coated ormosil suspensions prepared from both MSQ gels synthesized in DMSO and methanol. It can be clearly observed that the gels prepared in DMSO resulted in homogeneous films, without macropores (Fig. 3a). However, gels prepared in methanol resulted in films with macropores that scatter the light and result in translucent appearance (Fig. 3b). The thickness and refractive index of the films were measured by using spectroscopic ellipsometry in the reflection mode. Ellipsometry is, in general, a noninvasive optical characterization tool that is used to indirectly determine optical and electronic properties of thin films from the distinct reflections of the two polarization states of the incident light. From the measured $\Psi$ and $\Delta$ values and a physically appropriate model with variable parameters such as the complex index of refraction, film thickness, surface roughness, porosity is used to generate the measured data by fitting the variable parameters. Fig. 4 shows ellipsometry results for the D15 film. Two incidence angles (60 and 65) are used to obtain spectroscopic ellipsometry signals between 400 and $1000 \mathrm{~nm}$. Below $400 \mathrm{~nm}$, the UV absorption onset begins resulting in noisy data. In Fig. 4a, experimental data and generated Cauchy $n(\lambda)=A+\left(B / \lambda^{2}\right)+\left(C / \lambda^{4}\right)$ model data fit reasonably well. We
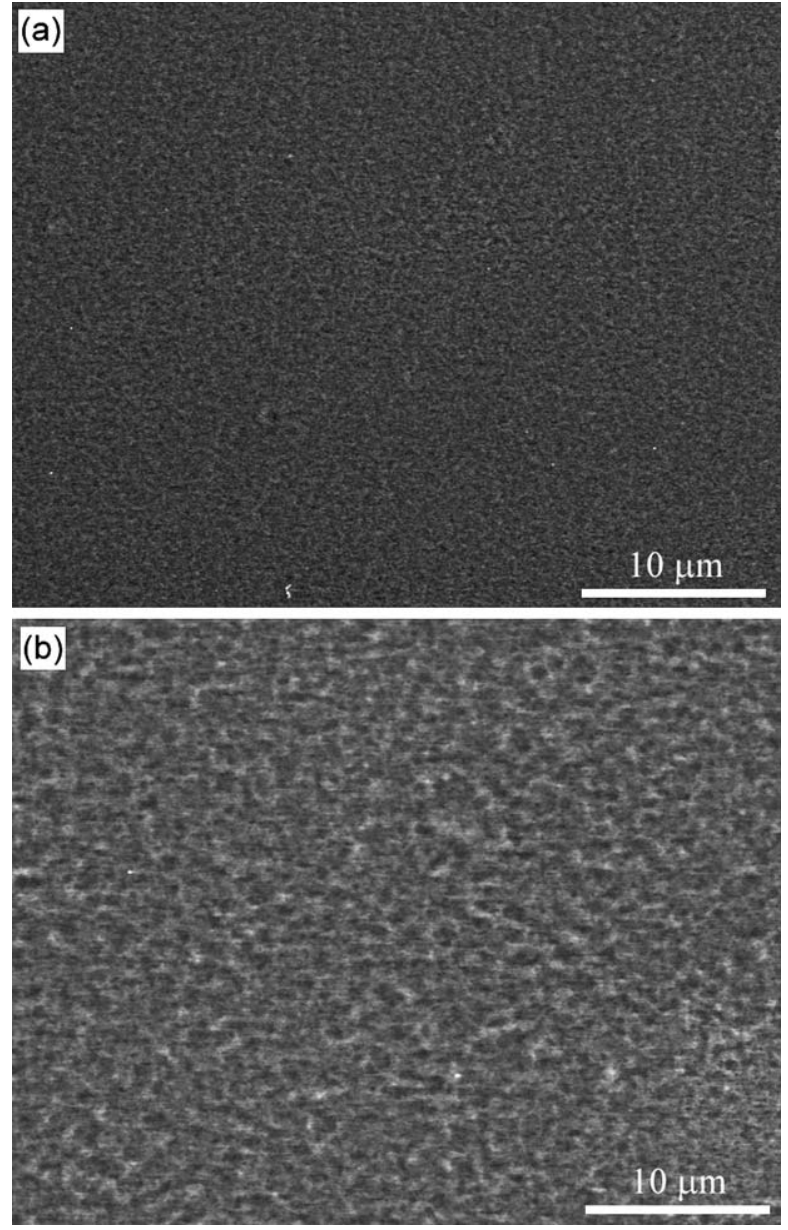

Fig. 3 SEM images of ormosil thin films, prepared from (a) gels in DMSO, and (b) in methanol. For DMSO gels, homogeneous film formation without macropores can be observed whereas for the methanol case macropores are observed.

used the simple Cauchy model since the ormosil film is transparent, i.e. $k(\lambda)=0$ in this band for the porous film layer on the glass substrate. We explored the effect of surface roughness by adding a surface roughness layer to the model which did not improve the already fine fitting between the measured and generated data. Fig. $4 \mathrm{~b}$ shows the measured refractive index of $\mathrm{D} 15$, as a function of wavelength, reaching as low as 1.13 . The porosities of the films were not calculated using ellipsometric models, but rather using the refractive index porosity equation, i.e. by comparing the porous film with a nonporous reference MTMS based thin film with a refractive index of 1.399. ${ }^{29,31}$ The refractive index and porosities of the prepared films are shown with respect to MTMS concentrations (Fig. 5a). The change in refractive indices (1.14-1.28) against MTMS concentrations is linear, which decreases with decreasing MTMS concentrations. Similarly, porosity is inversely proportional to the monomer concentration between 29 and $65 \%$. The thickness of the films produced from the D7 suspension is presented in Fig. 5b. The thickness of the films can be controlled by diluting the prepared suspensions. To show that we prepared a starting suspension by adding $5 \mathrm{ml}$ of methanol on the D7 and gel we diluted this suspension to get the desired concentration. Diluting the 

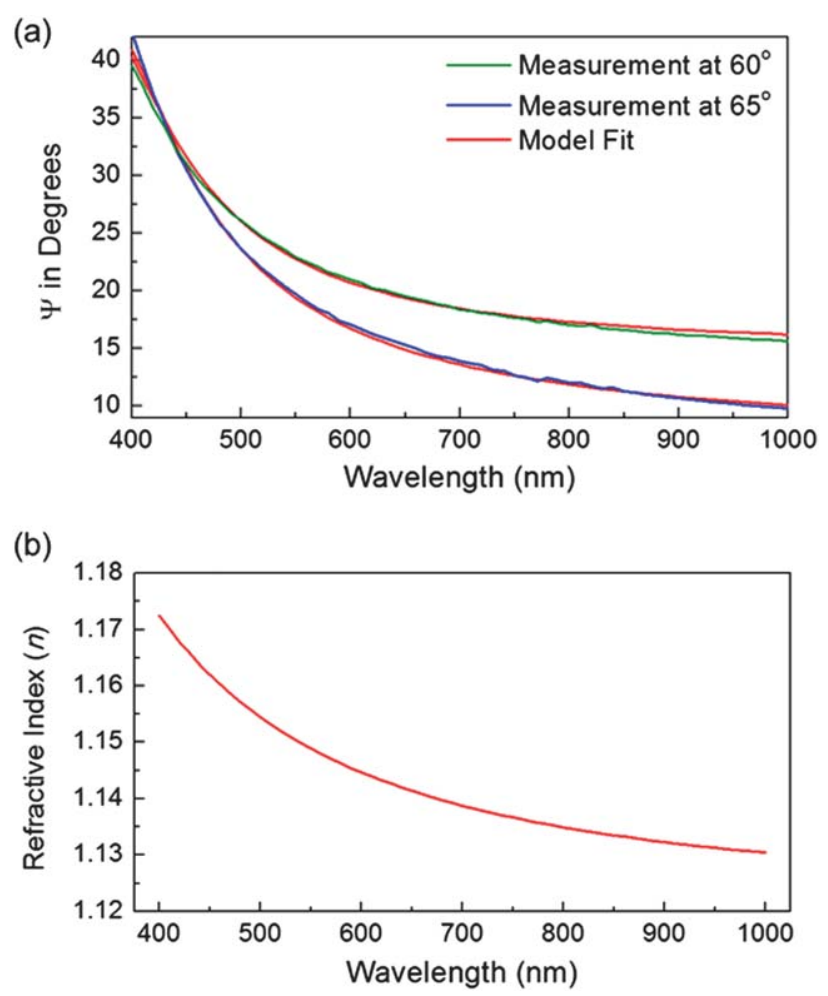

Fig. 4 Spectroscopic ellipsometry results for the D15 film. (a) Experimental and generated model data fit well with each other. (b) Measured refractive index of the D15 film as a function of the wavelength. The film exhibits very low refractive index reaching 1.13.

suspension decreases the film thickness and we obtained homogeneous ormosil films, as thin as $87 \mathrm{~nm}$. The thickness versus concentration is linear between 87 and $180 \mathrm{~nm}$. For thicker films, the thickness is higher than expected, due to the high viscosity of the concentrated suspensions. It was observed that diluting the suspension causes viscosity to decrease dramatically. The highest thickness obtained in this work is about $340 \mathrm{~nm}$. To obtain thicker films the suspensions can be spin coated repeatedly or spray coated for long times.

\section{Stable antireflective property of films}

Back reflection from optical surfaces is undesirable because it usually decreases the device performance. Antireflective coatings are routinely designed to enhance the light transmission through these surfaces. ${ }^{15,32-42}$ However, antireflective coatings on flexible substrates are still rare. ${ }^{32}$ An antireflective coating is based on destructive interference between the reflected light from the air/ film and the substrate/film interfaces. ${ }^{34}$ To achieve destructive interference between these interfaces, two conditions must be met. First, the refractive index ( $n$ ) of the film must be selected as $n_{\mathrm{c}}=\sqrt{n_{\mathrm{a}} n_{\mathrm{s}}}$, where $n_{\mathrm{c}}, n_{\mathrm{o}}$ and $n_{\mathrm{s}}$ are refractive indices of coating, air and substrate respectively, according to Fresnel equations to achieve minimum reflection. For example, for glass substrates, the refractive index should be selected as $\sim 1.23$. However, the lowest refractive index for homogeneous materials is around 1.35. To realize such low refractive indices, air must be introduced into the film as a second phase by generating pores to
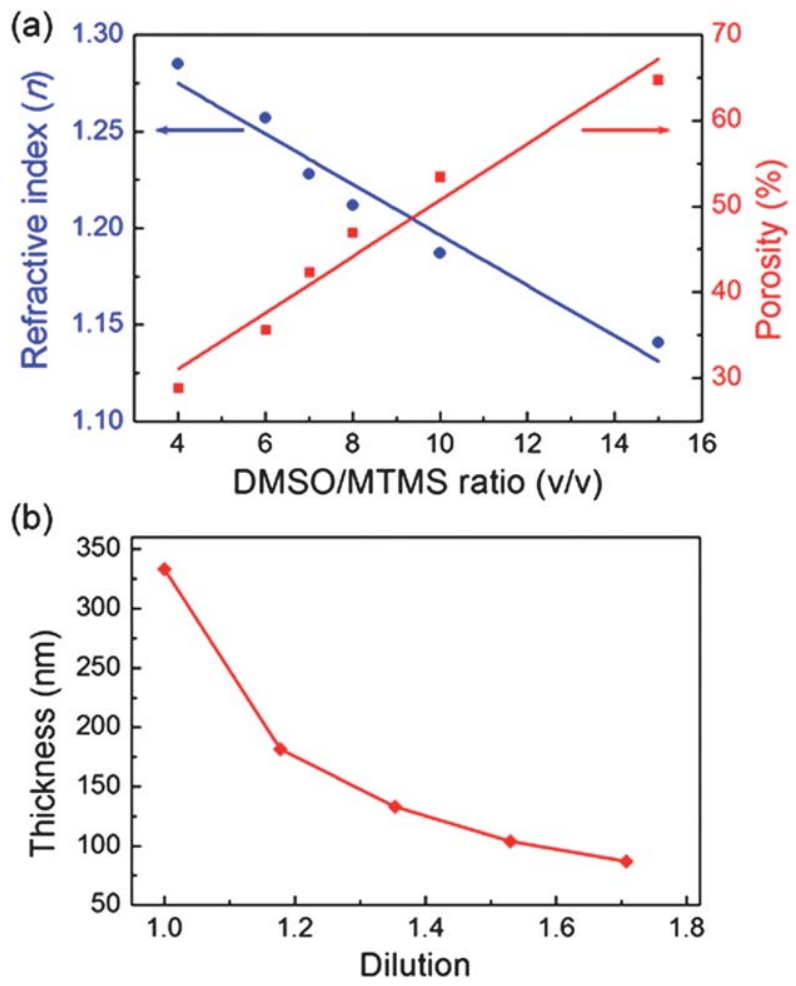

Fig. 5 (a) Effect of monomer concentration on refractive index and porosity. The porosity increases with decreasing monomer concentration. (b) Effect of ormosil sol dilution on spin coated film thicknesses. The films as thin as $\sim 90 \mathrm{~nm}$ were obtained by diluting the sol.

decrease the effective $n$ of the film. Also pore sizes must be smaller than $1 / 10$ of the wavelength of the light (which must be $<60 \mathrm{~nm}$ for visible light) to reduce Rayleigh scattering from the pores. Second, the thickness of the films must be a quarter of the effective wavelength (for visible wavelength thickness must be $\sim 100 \mathrm{~nm}$ ) to achieve maximum transmission. The nanoporous ormosil films produced in this work are good candidates as antireflective coatings on a variety of substrates including flexible ones, with their tunable thickness and refractive index, flexibility and durability.

The randomly distributed nanoporous structure of the D7 film on the glass substrate is shown in Fig. 6a. The pore size is smaller than $50 \mathrm{~nm}$, which is small enough to prevent light scattering. The side view of a $110 \mathrm{~nm}$ thick film coated on the silicon substrate is shown in Fig. 6b. The film has good thickness uniformity. The atomic force microscopy (AFM) image of D7 shows that the surface involves spherical bumps mostly smaller than $10 \mathrm{~nm}$ (Fig. 6c and d). Based on AFM characterization an average roughness was calculated to be $4.8 \mathrm{~nm}$ which is low enough to prevent light scattering from the surface. To demonstrate antireflective properties, we coated glass substrates with $\sim 110 \mathrm{~nm}$ thick D7 film $(n=1.23)$. Half of a glass slide was coated on both sides with D7 by spin coating (Fig. 7a). As expected, the coated part of the glass slide reflects less light because of the antireflective D7 film. Fig. 7b reveals the transmission spectra of D7 coated on both sides and bare glass slides. The D7 coated glass slide transmits $98.4 \%$ of light at $500 \mathrm{~nm}$ whereas uncoated glass slide transmits $91.3 \%$ of light (Fig. $7 b$ ). 

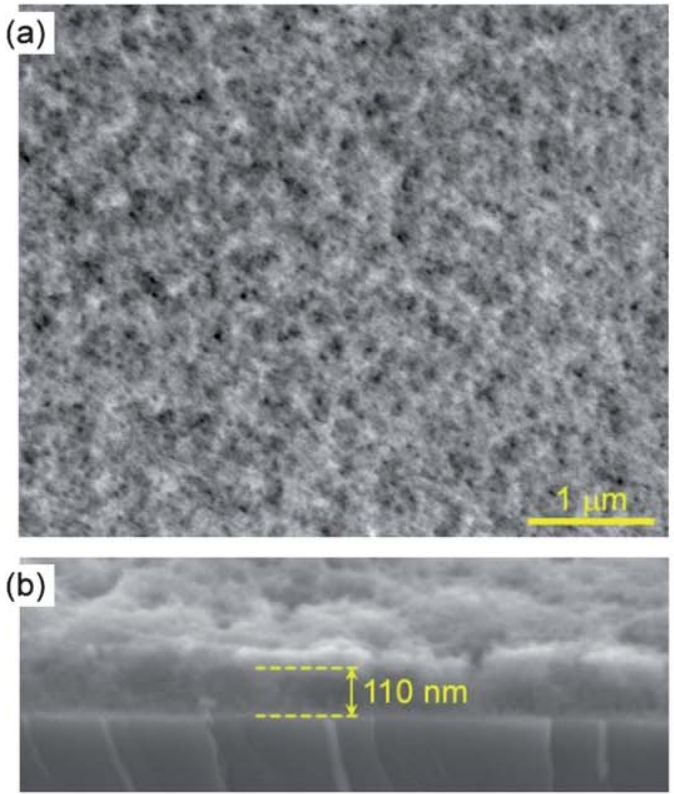

(c)

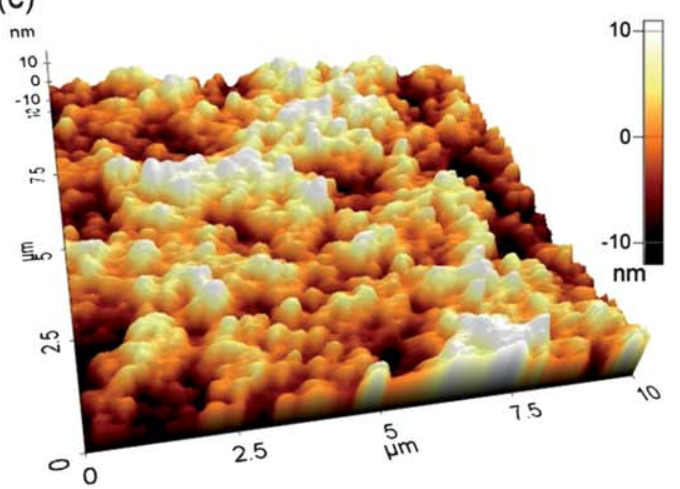

(d)

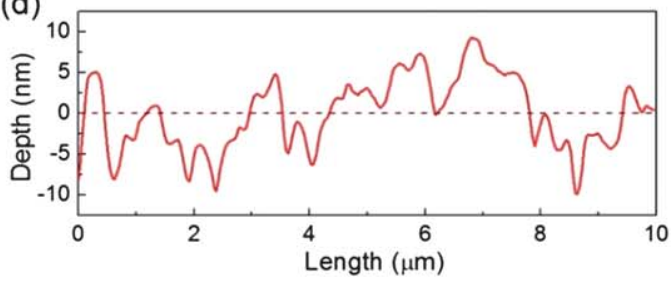

Fig. 6 (a) SEM image of the nanoporous D7 film on the glass substrate. (b) Side view of the $110 \mathrm{~nm}$ thick D7 film on the silicon substrate. (c) 3D AFM image of D7. (d) Depth profile obtained from the AFM image Based on the AFM images the surface roughness of the D7 film is calculated as $4.3 \mathrm{~nm}$.

Antireflective coating on the flexible substrate was demonstrated by spin coating the D7 suspension on both sides of the CA substrate. Fig. 8a shows a bent CA substrate coated with D7. From the image it can be clearly observed that the coated parts of the CA substrate reflect less light compared to the uncoated parts. To demonstrate the mechanically stable antireflective properties of nanoporous ormosil films, the D7 coated CA substrate was bent $180^{\circ}$ for 100 cycles (bending radius is $2.5 \mathrm{~mm}$ ). Visible light transmission spectra of the CA substrate, the D7 coated CA substrate and the D7 coated CA substrate after 100 bending cycles are shown in Fig. 8b. For the uncoated CA (a)

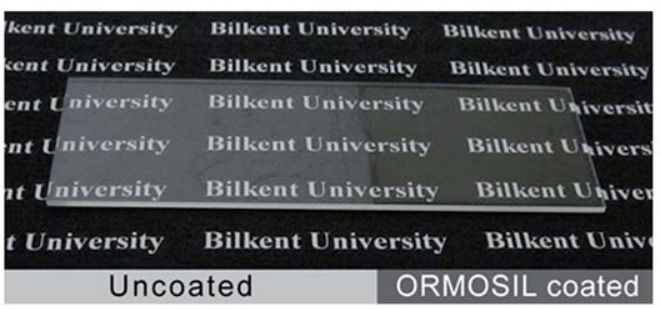

(b)

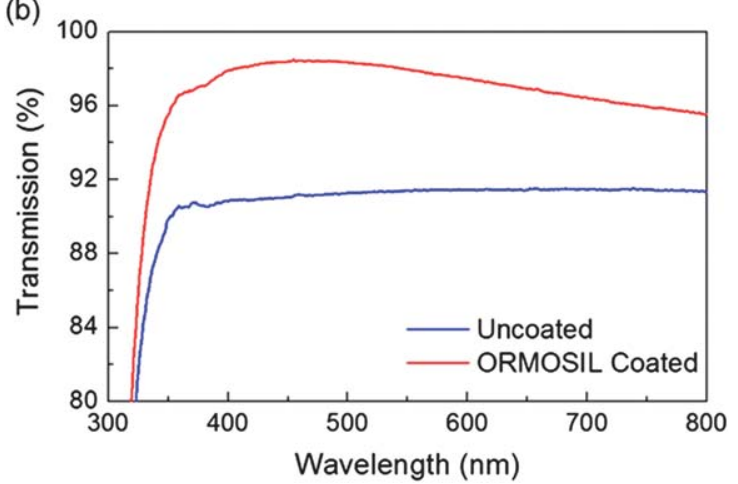

Fig. 7 (a) Photograph of partially D7 coated glass slide on both sides. Coated parts reflect less light indicating anti-reflective property. (b) Transmission spectra of spin coated D7 on both sides of the glass substrate (red) and the bare glass substrate (blue).

substrate the transmission maximum is $87.8 \%$ and for the coated one is $95.8 \%$ at $600 \mathrm{~nm}$. After 100 bending cycles, the transmission spectrum remains intact. The CA substrate does not allow excessive bending of the ormosil films, because the substrate itself deforms at bending radii below $2.5 \mathrm{~mm}$. Thus, for further bending, we used $10 \mu \mathrm{m}$ thick PEI as substrate and coated it with the D7 film (Fig. 8c). Although, the PEI substrate is not very transparent at visible wavelengths (transmission maximum is $68.5 \%$ ), it is suitable to test the flexibility of films at smaller bending radii. After D7 coating transmission of the PEI substrate increased to $\sim 78 \%$ and the transmission spectrum remains intact (Fig. 8d) as in the CA substrate, after 100 cycles of bending, but this time with a bending radius of $\sim 1 \mathrm{~mm}$. These results indicate good optical and mechanical flexibility of ormosil coatings on plastic substrates. To further investigate the mechanical stability of the ormosil surfaces, we performed a mechanical scratching test by using sandpaper. ${ }^{43}$ D7 and M7 films were scratched with sandpaper by applying approximately $10 \mathrm{kPa}$ pressure. After the scratching test the surface morphologies were investigated by SEM (Fig. S1, ESI $\dagger$ ) which revealed that most of the coating was removed by sandpaper for both D7 and M7 films, where they were in contact with sandpaper. Although the films are mechanically flexible, due to their high porosity and ultralow thickness, they are found not to be resistant to mechanical scratching.

\section{Hydrophobicity of nanoporous films}

The hydrophobicity of porous materials is important in terms of stability because the atmospheric humidity accumulates in the pores and results in pore collapse due to capillary stress developed in the pores. For example, in hydrophilic mesoporous silica 
(a)

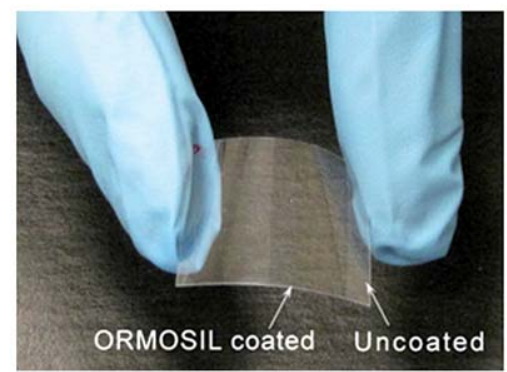

(b)

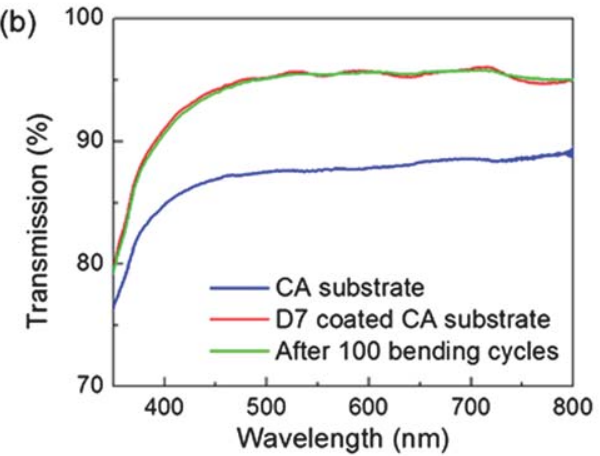

(c)

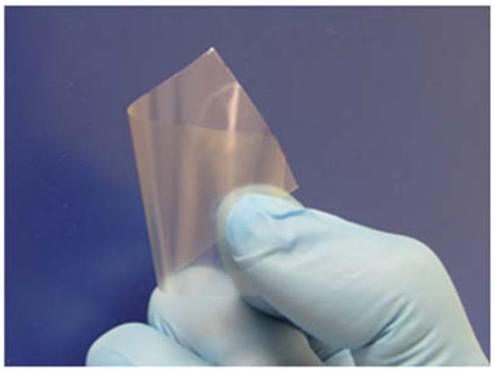

(d)

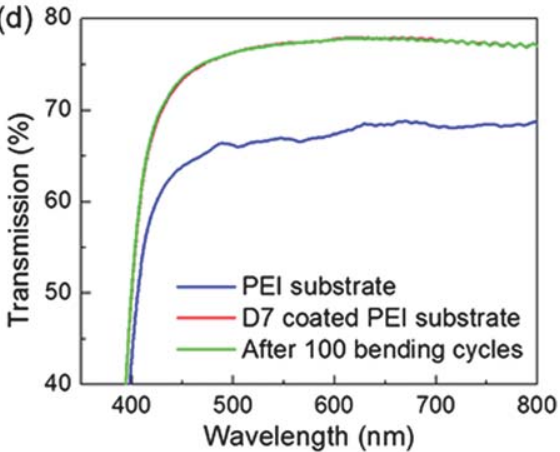

Fig. 8 (a) The D7 film coated on the bent CA substrate. Coated parts reflect less light indicating antireflective property. (b) Transmission spectra of uncoated, D7 coated on both sides of the CA substrate and spectra after 100 bending cycles (bending radius is $2.5 \mathrm{~mm}$ ). (c) Bent D7 film coated PEI substrate. (d) Transmission spectra of uncoated, D7 coated on both sides of the PEI substrate and spectra after 100 bending (bending radius is 1 mm). There is no significant change in transmission spectra after bending cycles for both films on CA and PEI substrates.

films, pore collapse may occur due to the atmospheric humidity. The films produced in this work are very hydrophobic with contact angles around $123^{\circ}$ on smooth polymer or glass substrates. However, this contact angle value is lower than the contact angles of previously reported ${ }^{23}$ films (reaching $179.9^{\circ}$ ) prepared from the phase separated MSQ gels. This is because of the lower surface roughness of the films $(4.8 \mathrm{~nm})$ compared to the previously reported films (reaching $120 \mathrm{~nm}$ ). Still films exhibit significant hydrophobicity due to the methyl groups covering the particle surface. ${ }^{23}$ The surface chemical groups of the D7 film were identified by FTIR spectroscopy and XPS. The absorption peaks of $\mathrm{Si}-\mathrm{C}\left(780 \mathrm{~cm}^{-1}\right)$ and $\mathrm{C}-\mathrm{H}\left(1280 \mathrm{~cm}^{-1}\right.$ and $\left.2975 \mathrm{~cm}^{-1}\right)$ bonds were observed in the FTIR spectrum (Fig. S2a, ESI $\dagger$ ). Surface hydroxyl groups $(-\mathrm{OH})$ can also be present in MSQ gels due to incomplete condensation of the monomers. ${ }^{23}$ However, no significant $\mathrm{O}-\mathrm{H}$ absorption peaks were observed in the FTIR spectrum of $\mathrm{D} 7$, indicating a high condensation rate of the MTMS monomer in DMSO. Also the XPS spectrum (Fig. S2b, ESI $\dagger$ ) revealed a high carbon content of $19 \%$. These results prove that the surface of the D7 film is mostly covered by methyl groups which give the hydrophobic behavior to the films. The hydrophobic nanopores of the ormosil films are expected to prevent the water accumulation and result in a durable pore structure.

The thermal stability of the hydrophobic D7 surface was investigated by heating the films to elevated temperatures (Fig. S3, ESI $\dot{\dagger}$ ). The surface is found to be thermally stable up to $500{ }^{\circ} \mathrm{C}$. In this region, the water contact angle of the film raised slightly to $133^{\circ}$ which may be because of the increase in the surface roughness after heat treatment. ${ }^{23}$ After $500{ }^{\circ} \mathrm{C}$ the contact angle of the film decreases sharply with increasing (a)
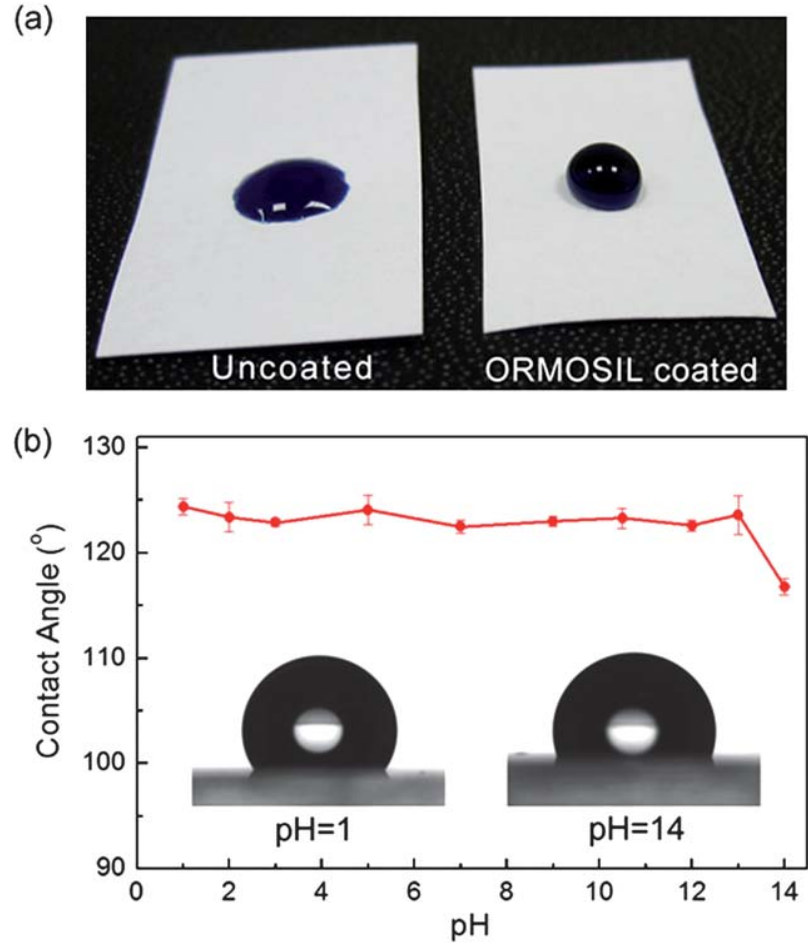

Fig. 9 (a) Water droplet on bare paper and D7 coated substrates. Water droplet on uncoated paper is absorbed. On the other hand, the water droplet coated on paper stands with a near spherical shape. (b) Static contact angle change of the D7 film on the glass substrate with $\mathrm{pH}$. The ormosil films exhibit good durability over a wide $\mathrm{pH}$ range. 
temperature and it reaches $\sim 5^{\circ}$ at $600{ }^{\circ} \mathrm{C}$. The decrease in the contact angle is due to the decomposition of the surface methyl groups. The FTIR and XPS spectra of the D7 film after calcination at $600{ }^{\circ} \mathrm{C}$ (Fig. S2, ESI $\dagger$ ) show the sharp decrease in the surface methyl groups and increase in hydroxyl groups. In the FTIR spectrum the intensity of $\mathrm{Si}-\mathrm{C}$ and $\mathrm{C}-\mathrm{H}$ absorption peaks was reduced significantly. On the other hand, $\mathrm{O}-\mathrm{H}$ absorption peaks $\left(3460 \mathrm{~cm}^{-1}\right.$ and $\left.1640 \mathrm{~cm}^{-1}\right)$ became apparent. Also XPS analysis revealed a very low carbon content of 3.3\% and a high oxygen content of $52.7 \%$ (38.7\% before calcination). Replacing the methyl groups with hydroxyl groups resulted in a hydrophilic surface.

To demonstrate the wide applicability of the colloidal ormosil suspensions, we deposited a D7 suspension with drop casting on a paper substrate. The hydrophobic D7 film gives the paper resistance against water. Uncoated paper is highly hydrophilic and absorbs water quickly. Nanoporous ormosil coated paper shows highly hydrophobic behavior with a contact angle reaching $143^{\circ}$ (Fig. 9a). The higher contact angle observed on the paper substrate is due to the greater surface roughness of the paper. Also, we observed that the hydrophobicity of the paper substrate is stable and there is no significant change in the contact angle even after 25 cycles of bending (bending radius is $2.5 \mathrm{~mm}$ ). Fig. $9 \mathrm{~b}$ shows the contact angle of the films is stable over the whole $\mathrm{pH}$ range $(\mathrm{pH} 1-14)$. There is a slight decrease $\left(6^{\circ}\right)$ in the contact angle at $\mathrm{pH} 14$ but still the surface is hydrophobic with a contact angle of $117^{\circ}$.

\section{Conclusion}

In conclusion, we described a novel method for the production of flexible nanoporous ormosil films at room temperature and neutral $\mathrm{pH}$ conditions. Films were deposited from colloidal suspensions with common thin film deposition methods. The colloidal suspensions are stable for several months and offer great reproducibility and simplicity for producing nanoporous and stable thin films on a variety of substrates. We prepared several ormosil thin films on glass, polymer, silicon and paper substrates. The films on flexible substrates reveal durable antireflective and surface properties after many cycles of bending. We believe that our novel production method of nanoporous flexible ormosil thin films on sensitive substrates may expand their use in low cost electronic and optical devices and lab-onpaper applications.

\section{Acknowledgements}

We thank Hakan Deniz for fruitful discussions and Mustafa Güler for TEM images. This work is supported by TÜBITAK under the Project No. 106G090 and 110M412. MB acknowledges support from the Turkish Academy of Sciences Distinguished Young Scientist Award (TUBA GEBIP).

\section{References}

1 M. Pagliaro, R. Ciriminna and G. Palmisano, J. Mater. Chem., 2009, 19, 3116.

2 H. S. Lim, J. H. Baek, K. Park, H. S. Shin, J. Kim and J. H. Cho, $A d v$. Mater., 2010, 22, 2138.

3 Y. Tamar and D. Mandler, Electrochim. Acta, 2008, 53, 5118.
4 M. J. Mosquera, D. M. Santos and T. Rivas, Langmuir, 2010, 26, 6737.

5 P. G. Parejo, M. Zayat and D. Levy, J. Mater. Chem., 2006, 16, 2165. 6 B. Mahltig, C. Swaboda, A. Roesslerc and H. Bottcher, J. Mater. Chem., 2008, 18, 3180.

7 P. Baglioni, L. Dei, E. Carretti and R. Giorgi, Langmuir, 2009, 25, 8373.

8 Y. Tang, J. A. Finlay, G. L. Kowalke, A. E. Meyer, F. V. Bright, M. E. Callow, J. A. Callow, D. E. Wendt and M. R. Detty, Biofouling, 2005, 21, 59.

9 J. L. Defreese and A. Katz, Chem. Mater., 2005, 17, 6503.

10 H. C. Kim, G. Wallraff, C. R. Kreller, S. Angelos, V. Y. Lee, W. Volksen and R. D. Miller, Nano Lett., 2004, 4, 1169.

11 B. J. Scott, G. Wirnsberger and G. D. Stucky, Chem. Mater., 2001, 13, 3140.

12 H. L. Castricum, G. G. Paradis, M. C. Mittelmeijer-Hazeleger, R. Kreiter, J. F. Vente and J. E. ten Elshof, Adv. Funct. Mater., 2011, 21, 2314.

13 J. Wan, K. Qian, J. Zhang, F. Liu, Y. Wang, P. Yang, B. Liu and C. Yu, Langmuir, 2010, 26, 7444.

14 P. Yang, G. Wirnsberger, H. C. Huang, S. R. Cordero, M. D. McGehee, B. Scott, T. Deng, G. M. Whitesides, B. F. Chmelka, S. K. Buratto and G. D. Stucky, Science, 2000, 287, 465.

15 S. Kim, J. Cho and K. Char, Langmuir, 2007, 23, 6737.

16 N. J. Trujillo, Q. Wu and K. K. Gleason, Adv. Funct. Mater., 2010, 20, 607.

17 T. Asefa, M. J. MacLachan, N. Coombs and G. A. Ozin, Nature, 1999, 402, 867.

18 H. C. Kim, J. B. Wilds, C. R. Kreller, W. Volksen, P. J. Brock, V. Y. Lee, T. Magbitang, J. L. Hedrick, C. J. Hawker and R. D. Miller, Adv. Mater., 2002, 14, 1637.

19 L. Hu, H. Wu, F. L. Mantia, Y. Yang and Y. Cui, ACS Nano, 2010, 4, 5843.

20 S. R. Forrest, Nature, 2004, 428, 911.

21 K. Jo, T. Lee, H. J. Choi, J. H. Park, D. J. Lee, D. W. Lee and B. S. Kim, Langmuir, 2011, 27, 2014.

22 S. S. Prakash, C. J. Brinker, A. J. Hurd and S. M. Rao, Nature, 1995, 374, 439.

23 H. Budunoglu, A. Yildirim, M. O. Guler and M. Bayindir, ACS Appl. Mater. Interfaces, 2011, 3, 539.

24 K. Kanamori, M. Aizawa, K. Nakanishi and T. Hanada, Adv. Mater., 2007, 19, 1589.

25 K. Kanamori, K. Nakanishi, K. Hirao and H. Jinnai, Langmuir, 2003, 19, 5581 .

26 H. Dong, M. A. Brook and J. D. Brennan, Chem. Mater., 2005, 17, 2807.

27 S. D. Bhagat, C. S. Oh, Y. H. Kim, Y. S. Ahn and J. G. Yeo, Microporous Mesoporous Mater., 2007, 100, 350.

28 A. C. Pierre and G. M. Pajonk, Chem. Rev., 2002, 102, 4243.

29 A. Yildirim, H. Budunoglu, H. Deniz, M. O. Guler and M. Bayindir, ACS Appl. Mater. Interfaces, 2010, 2, 2892.

30 J. Kobler, K. Moller and T. Bein, ACS Nano, 2008, 2, 791.

31 G. S. Kim and S. H. Hyun, Thin Solid Films, 2004, 460, 190

32 Z. Wu, J. Walish, A. Nolte, L. Zhai, R. E. Cohen and M. F. Rubner, Adv. Mater., 2006, 18, 2699.

33 S. Walheim, E. Schaffer, J. Mlynek and U. Steiner, Science, 1999, 283, 520.

34 P. Podsiadlo, L. Sui, Y. Elkasabi, P. Burgardt, J. Lee, A. Miryala, W. Kusumaatmaja, M. Carman, R. M. Shtein, J. Kieffer, J. Lahann and N. A. Kotov, Langmuir, 2007, 23, 7901.

35 M. Ibn-Elhaj and M. Schadt, Nature, 2001, 410, 796.

36 X. Li, J. Gao, L. Xue and Y. Han, Adv. Funct. Mater., 2010, 20, 259.

37 M. Manca, A. Cannavale, L. De Marco, A. S. Arico, R. Cingolani and G. Gigli, Langmuir, 2009, 25, 6357.

38 Z. C. Sun, F. Bai, H. M. Wu, S. K. Schmitt, D. M. Boye and H. Y. Fan, J. Am. Chem. Soc., 2009, 131, 13594.

39 Y. Du, L. E. Luna, W. S. Tan, M. F. Rubner and R. E. Cohen, $A C S$ Nano, 2010, 4, 4308.

40 X. Li, X. Du and J. He, Langmuir, 2010, 26, 13528.

41 J. Hiller, J. D. Mendelsohn and F. M. Rubner, Nat. Mater., 2002, 1, 59.

42 L. N. Nagy, N. Abraham, Ö. Sepsi, E. Hild, D. Cot, A. Ayral and Z. Horvölgyi, Langmuir, 2008, 24, 12575.

43 L. Yang, L. Yong and J. Sun, Angew. Chem., Int. Ed., 2010, 49, 6129. 